\title{
离子极化和离子水化能
}

\author{
温元 凯 \\ （中国科学技术大学化学系) \\ 郡俊 \\ (浙江平阳矾矿中学)
}

近年来,随着离子交换、离子选择性电极 和电渗析等新技术的发展, 离子在水溶液中 的行为正日益受到重视和研究, 离子水化能 就是其中重要的方面之一 ${ }^{[1-3]}$.

离子水化能是气态离子溶于大量水中成 为无限稀释溶液时释放的能量, 即反应

$$
M^{n+}+a q .=M^{n+}(a q .)
$$

的反应热, 水化能在无机化学、物理化学等方 面都有不少应用。

按照 Born $^{[4]}$ 的理论,离子的水化能可由 下列方程表示:

$$
L=\frac{N e Z^{2}}{2 r}\left[1-\frac{1}{D}+\frac{T}{D^{2}}\left(\frac{\partial D}{\partial T}\right)\right],
$$

此处, $N$ 是 Avogadro 常数; $e$ 是电子电荷; $D$ 是介电常数; $r$ 是离子半径再加水分子的直 径; $Z$ 是离子电荷. 但是,式 (1) 后被证明并 不适用于计算离子的水化能 ${ }^{[5]}$. 经典静电理 论并不符合实际情况. 用量子化学或分子轨 道理论计算离子水化能目前只能对极少数离 子进行, 而且也还存在不少困难 ${ }^{[6,7]}$. 因此, 有 人建议用经验公式来计算离子的水化能 ${ }^{[8,9]}$. 比较简单的如 ${ }^{[20]}$ :

$$
L_{i}=\frac{165.5 \mathrm{Z}^{2}}{r+l},
$$

式中 $r$ 为离子半径, $z$ 是离子电荷, $l$ 是一常 数,对阴离子为 0.4 , 对阳离子为 0.8 . 式 (2) 计算的离子水化能对惰气构型离子与实验值 粗略符合. 然而, 倘若我们应用式 (2) 于非 惰气构型离子时, 则发现有很大的偏差, 离子 水化能的计算值皆明显偏低于实验值. 我们 认为, 这是由于忽略了离子极化作用引起的.
离子和水分子之间，除了一般的静电相互作 用外, 还存在有离子极化作用, 这种极化作 用使水分子的电子云发生变形而导致键合加 强, 引起离子和水分子间结合能即水化能升 高. 因此, 根据解决非标准型离子晶体晶格 能问题时采用的方法 ${ }^{[1,12]}$, 建议把离子的真 实水化能表达为纯离子型水化能和离子水化 极化能两部分之和:

$$
L=L_{i}+L_{p},
$$

其中纯离子型水化能 $L_{i}$ 可从式 (2) 计算. 至 于水化极化能 $L_{p}$, 根据我们对离子晶体中离 子极化能的研究, 得知离子极化能可表达为 阳离子极化力和阴离子变形性的函数. 运用 这个规律于离子水化作用中, 因被极化的对 象已固定为水分子. 所以, 离子水化极化能 就只与阳离子的极化力有关. 据此, 本文提 出一个计算非惰气构型离子水化极化能的经 验公式:

$$
L_{p}=a \frac{Z^{*_{2}}}{r}+b,
$$

式中 $Z^{*} / r$ 是我们提出的衡量阳离子极化力 的标度 ${ }^{[1]}, Z^{*}$ 是阳离子的有效核电荷, $r$ 是 离子半径. $a$ 和 $b$ 是经验常数, 视离子价数而 定,一价离子为 1.3 和 -15 ; 二价离子为 0.8 . 和 31 ; 三价离子为 1.7 和 -46 ; 四价离子为 0.3 和 71 .

利用式(4)及式(2), 我们计算了 31 种非 情气构型阳离子的水化能, 其结果列于表 1 中. 其中 $L_{\text {* }}$ 取自 Basolo 和 Pearson ${ }^{[3]}$ 的汇集. 为了便于比较, 我们把马登勇 ${ }^{[21]}$ 应用离子能

本文 1975 年 4 月 21 日收到。 
表 1 非惰气构型阳离子的水化能(千卡/克分子)

\begin{tabular}{|c|c|c|c|c|c|c|c|c|}
\hline \multirow{2}{*}{ 子 } & \multirow{2}{*}{$\frac{Z^{* 2}[11]}{r}$} & \multirow{2}{*}{$L_{\text {实能 }}^{[13]}$} & \multirow{2}{*}{$L_{i}$} & \multirow{2}{*}{$L_{p}$} & \multicolumn{2}{|c|}{ 本文 计 算 } & \multicolumn{2}{|c|}{ 马登勇 ${ }^{[14]}$ 的计算 } \\
\hline & & & & & $L_{\text {计 }}$ & $\Delta$ & $L_{\text {歹 }}$ & $\Delta$ \\
\hline $\mathrm{Cu}^{+}$ & 42.6 & 139 & 95 & 39 & 134 & +5 & 125 & +14 \\
\hline $\mathrm{Ag}^{+}$ & 39.7 & 116 & 86 & 34 & 120 & -4 & 114 & -2 \\
\hline $\mathrm{Au}^{+}$ & 71.0 & 154 & 79 & 76 & 155 & -1 & 114 & +40 \\
\hline $\mathrm{Tl}+$ & 26.7 & 80 & 72 & 20 & 92 & -12 & 90 & -10 \\
\hline $\mathrm{Ti}^{2+}$ & 12.7 & 446 & 401 & 41 & 442 & +4 & 427 & +19 \\
\hline $\mathrm{V}^{2+}$ & 19.7 & 453 & 406 & 46 & 452 & +1 & 441 & +12 \\
\hline $\mathrm{Cr}^{2+}$ & 25.8 & 460 & 411 & 52 & 463 & -3 & 463 & -3 \\
\hline $\mathrm{Mn}^{2+}$ & 30.3 & 445 & 387 & 55 & 442 & +3 & 440 & +5 \\
\hline $\mathrm{Fe}^{2+}$ & 41.9 & 468 & 406 & 65 & 471 & -3 & 477 & -9 \\
\hline $\mathrm{Co}^{2+}$ & 52.3 & 497 & 409 & 73 & 482 & +15 & 479 & +18 \\
\hline $\mathrm{Ni}^{2+}$ & 66.5 & 507 & 419 & 84 & 503 & -4 & 494 & +13 \\
\hline $\mathrm{Cu}^{2+}$ & 77.0 & 507 & 414 & 93 & 507 & 0 & 525 & -18 \\
\hline $\mathrm{Zn}^{2+}$ & 64.2 & 492 & 406 & 82 & 488 & +4 & 494 & -2 \\
\hline $\mathrm{Cd}^{2+}$ & 57.6 & 437 & 362 & 75 & 437 & 0 & 437 & 0 \\
\hline $\mathrm{Hg}^{2+}$ & 105.3 & 441 & 345 & 110 & 455 & -14 & 444 & -3 \\
\hline $\mathrm{Sn}^{2+}$ & 16.3 & 374 & 336 & 48 & 384 & -10 & 388 & -14 \\
\hline $\mathrm{Pb}^{2+}$ & 40.4 & 359 & 312 & 56 & 368 & -9 & 357 & +2 \\
\hline $\mathrm{Ag}^{2+}$ & 92.0 & 411 & 382 & 84 & 466 & -55 & 486 & -75 \\
\hline $\mathrm{Rh}^{2+}$ & 79.0 & 486 & 414 & 74 & 488 & -2 & 490 & -4 \\
\hline $\mathrm{Pd}^{2+}$ & 86.0 & 505 & 409 & 80 & 489 & +16 & 493 & +12 \\
\hline $\mathrm{V}^{3+}$ & 28.5 & 1053 & 1034 & 2 & 1036 & +17 & 1034 & +19 \\
\hline $\mathrm{Cr}^{3+}$ & 38.3 & 1105 & 1041 & 19 & 1060 & +45 & 1119 & -14 \\
\hline $\mathrm{Mn}^{3+}$ & 47.8 & 1098 & 1010 & 30 & 1040 & +58 & 1140 & +58 \\
\hline $\mathrm{Fe}^{3+}$ & 58.3 & 1072 & 1019 & 53 & 1072 & 0 & 1104 & -32 \\
\hline $\mathrm{Co}^{3+}$ & 74.4 & 1126 & 1041 & 86 & 1127 & -1 & 1154 & -28 \\
\hline $\mathrm{Ti}^{3+}$ & 19.3 & 1027 & 1060 & -13 & 1047 & -20 & 1071 & -44 \\
\hline $\mathrm{Ga}^{3+}$ & 99.7 & 1124 & 1056 & 88 & 1144 & -20 & 1163 & -37 \\
\hline $\mathrm{In}^{3+}$ & 82.3 & 995 & 871 & 143 & 1014 & -19 & 1006 & -11 \\
\hline $\mathrm{Tl}^{3+}$ & 133.9 & 984 & 810 & 156 & 966 & +18 & 919 & +65 \\
\hline $\mathrm{Ce}^{4+}$ & 26.5 & 1542 & 1463 & 79 & 1542 & 0 & & \\
\hline $\mathrm{Sn}^{4+}$ & 127.1 & 1827 & 1719 & 108 & 1827 & 0 & & \\
\hline
\end{tabular}

量半径的计算结果也列入了表 1. 从表中可 以看出,本文计算结果比马登勇的方法要好. 一些偏差特别大的离子如 $\mathrm{Ag}^{2+} 、 \mathrm{Mn}^{3+} 、 \mathrm{Pd}^{2+}$ 、 $\mathrm{Cr}^{3+}$, 与金属氢氧化物溶解度规律中的反常 类似 ${ }^{[15]}$ ，可能与这些离子在水溶液中的稳定 性、高度水解或聚合有关, 导致实验数据不甚 可靠,也可能是其离子半径还不确定的缘故.

\section{参考资 料}

(1] Rossinsky, D. R., Chem. Rev., 65 (1965), 467.

(2) Hunt, J. P., Metal lons in Aqueous Solution, New York, 1963.
[3] 大滰志, 化学 (日本), 26 (1971), 1179.

[4] Born, M., Z. Phys., 1 (1920), 45.

[5] 黄子蝍, 化学通报, $1961,12,625$.

[6] Бунятян, Б. Х., Теор. Эксп. Хим., 10 (1974), 233.

[7] Bange, K. \& Stogard, A., Acta Chem. Scand., 27 (1973), 2683.

[8] Voet, A., Trans. Faraday Soc., 32 (1936), 1301.

[9] Кондратьев, В. Н., и Соколов, Н. Д., Журн. Физ. Хим., 29 (1955), 1265.

[10] K. E. 亚奇米尔斯基,络合物热化学,科学出版社, 1959,41

[11] 温元凯、䂙俊,地球化学, $1973,4,276$.

[12] 温元凯、那俊,地球化学, $1975,1,35$.

[13] Basolo, F. \& Pearson, R. G., Mechanisms of Inorganic Chemistry, New York, 1958, 66.

[14] 马登勇,科学通报, $1965,6,544$.

[15] 温元凯、郡俊,科学通报, 20 (1975), 3, 131 . 